\title{
Stereotactic body radiotherapy for the treatment of spinal metastases
}

\author{
Ehsan H. Balagamwala • Sheen Cherian • \\ Lilyana Angelov • John H. Suh • Toufik Djemil • \\ Simon S. Lo • Arjun Sahgal • Eric Chang • Bin S. Teh • \\ Samuel T. Chao
}

Received: 17 May 2012 / Accepted: 31 May 2012 / Published online: 19 June 2012

(C) Springer-Verlag 2012

\begin{abstract}
Introduction Spine stereotactic body radiation therapy (sSBRT) allows for the delivery of a high dose of radiation to spine metastases while respecting the dose limits of the adjacent spinal cord. In contrast with conventional radiation, the dose to spine metastases is limited by the spinal cord tolerance since the spinal cord is in the treatment field. sSBRT allows for reirradiation of spinal metastases, as well as higher doses to be delivered particularly for radioresistant metastases. It is also being used post laminectomy and decompressive surgery as primary treatment for malignant spinal cord compression instead of conventional external beam radiation therapy. Although experience and evidence are growing, variations in practice remain.

Purpose We review the technical considerations and clinical applications of sSBRT.
\end{abstract}

\section{E. H. Balagamwala}

Cleveland Clinic Lerner College of Medicine,

Case Western Reserve University,

Cleveland, OH, USA

\section{S. Cherian · J. H. Suh $\cdot$ T. Djemil $\cdot$ S. T. Chao}

Department of Radiation Oncology, Taussig Cancer Institute,

Cleveland Clinic,

Cleveland, OH, USA

\section{Angelov}

Department of Neurological Surgery, Neurological Institute,

Cleveland Clinic,

Cleveland, OH, USA

L. Angelov $\cdot$ J. H. Suh $\cdot$ S. T. Chao $(\bowtie)$

Rose Ella Burkhardt Brain Tumor and Neuro-oncology Center,

Neurological Institute, Cleveland Clinic,

9500 Euclid Avenue,

Cleveland, OH 44195, USA

e-mail: Chaos@ccf.org
Keywords Spine metastasis · Stereotactic radiosurgery · Stereotactic body radiation therapy

\section{Introduction}

Stereotactic body radiation therapy (SBRT) is a novel radiation technique that delivers a high dose of radiation to the tumor with great precision, by taking advantage of recent advances in real-time tumor tracking and radiation dose delivery systems [1]. Metastasis to the spine occurs in up to $70 \%$ of all cancer patients, and 10 to $20 \%$ of cancer patients with bony spinal metastasis will develop symptomatic spinal cord compression [2]. Spinal metastases are commonly treated with a fractionated course of external beam radiation therapy

S. S. Lo

Department of Radiation Oncology, University Hospitals Seidman Cancer Center, Case Comprehensive Cancer Center,

Cleveland, $\mathrm{OH}$, USA

A. Sahgal

Department of Radiation Oncology, The Princess Margaret

Hospital and Sunnybrook Health Sciences

Center of the University of Toronto,

Toronto, ON, Canada

E. Chang

Department of Radiation Oncology,

University of Southern California,

Los Angeles, CA, USA

B. S. Teh

Department of Radiation Oncology, The Methodist Hospital, Houston, TX, USA 
(EBRT). However, if there is spinal cord compression or vertebral column instability, surgical decompression and spinal stabilization would precede EBRT. In some patients, surgical decompression is not possible due to short overall life expectancy, multiple levels of spinal metastasis, or preexisting medical conditions. In these patients, EBRT is the only available therapeutic option.

One of the main goals of palliative spinal radiotherapy is pain control, and conventional dose fractionated EBRT has a pain response rate of $60 \%$ [3]. Overall, $20 \%$ of patients previously treated with a conventional EBRT dose of 8 Gy in one fraction will require retreatment due to recurrence of pain. This is challenging because with conventional EBRT, the spinal cord within the treated field would have received a substantial dose of radiation, and a further palliative dose of radiotherapy is likely to exceed the spinal cord tolerance. This leads to retreatment with lower biologically effective doses of fractionated EBRT as the cumulative tolerance to the organ at risk (OAR), in this case the spinal cord, decides the dosefractionation schedule as opposed to tumor control [3].

SBRT delivers a high dose of radiation to the tumor and its vasculature, which can overcome any inherent tumor radioresistance to conventionally fractionated EBRT. Image-guided radiation therapy (IGRT) and intensity modulation radiation therapy (IMRT) have allowed the treated volume in SBRT to minimize dose to the spinal cord. Superior patient immobilization techniques and extreme hypofractionation which is inherent in SBRT, thus making such immobilization practical, has allowed the use of tighter margins. Retreatment pain control rates with SBRT are reported to be $65-85 \%$ [3]. The net result of spine SBRT (sSBRT) is the safe delivery of a very high biologically equivalent dose (BED) of radiation that is spinal cord sparing and may overcome any tumor radioresistance. Given the outcomes achieved with retreatment, it is now being used to treat patients up front, particular in patients with radioresistant histologies. The aim of this review is to provide an overview of the general principles and indications that guide sSBRT and also to discuss the local control rates with this novel radiation technique.

\section{Technical considerations}

Spine SBRT is a resource-intensive treatment modality for spinal tumors that utilizes the expertise of radiation oncologists, neurosurgeons, medical physicists, and radiation therapists in delivering precise high-dose radiation in a safe, convenient, and effective manner. Similar to radiosurgical techniques in the brain, SSBRT requires precision radiation delivery in the range of 1-2 mm [4]. To safely and effectively perform sSBRT, the following components are required: a body immobilization system, linear accelerator equipped with a multileaf collimator or circular collimator on robotic arms, a sophisticated treatment planning system with accurate delineation of target and organs at risk, and intrafraction image guidance.

\section{Body immobilization}

In sSBRT, the dose gradient is typically very steep outside the target volume in order to spare the spinal cord. Therefore, unlike extraspinal applications of SBRT, sSBRT requires a translational accuracy of $<2 \mathrm{~mm}$ and a rotational accuracy of $<2^{\circ}[5,6]$. Although respiration has a minimal impact on the motion of spinal tumors, rigid fixation of the spine is not readily achievable. Similar to fixation used for Gamma Knife radiosurgery, Hamilton et al. have described an invasive spinal fixation technique, which is not practical for sSBRT especially if a multifraction treatment plan is developed [7]. Therefore, many centers have utilized a near-rigid immobilization system. At the Cleveland Clinic, we utilize the Elekta BodyFIX stereotactic body frame (Medical Intelligence, Schwabmünchen, Germany) which consists of a carbon fiber base plate, whole-body vacuum cushion, vacuum system, and plastic fixation sheet for thoracic and lumbar lesions [8] (Figs. 1 and 2). The BodyFIX immobilization system is also in use at MD Anderson Cancer Center (MDACC) as well the University of Toronto and multiple other institutions $[4,9]$. Some institutions such as Memorial Sloan-Kettering Cancer Center (MSKCC) and the University of Heidelberg, Germany, have developed in-house systems for near-rigid immobilization. Hyde et al. recently evaluated their experience with BodyFIX immobilization system and cone beam computed tomography $(\mathrm{CBCT})$ imaging scan to evaluate setup error and intrafraction motion [10]. They studied 42 consecutive patients with thoracic or lumbar spinal metastases and found that patient positioning errors were relatively small $(90 \%$ were within $1 \mathrm{~mm}$

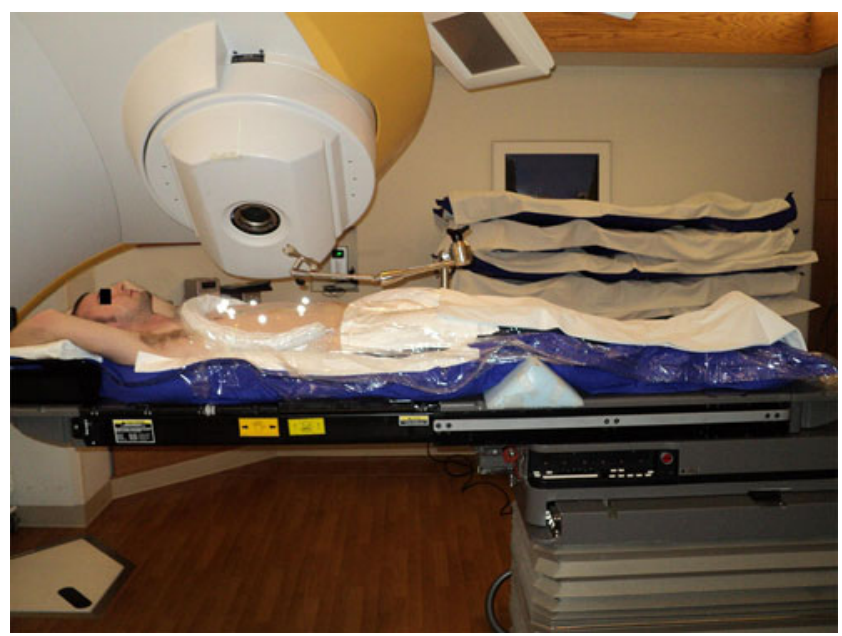

Fig. 1 The Elekta BodyFIX stereotactic body frame (Medical Intelligence, Schwabmünchen, Germany) which consists of a carbon fiber base plate, whole-body vacuum cushion, vacuum system, and plastic fixation sheet for thoracic and lumbar lesions 


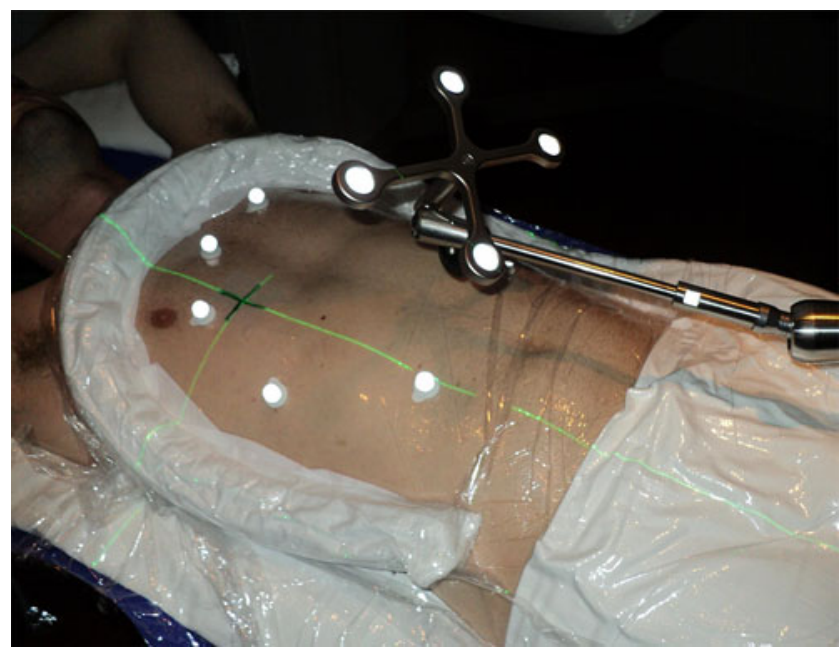

Fig. 2 Infrared fiducials placed on the BodyFIX to track patient motion and correct setup errors. For cervical spine SBRT, a conventional rigid five-point thermoplastic mask is used

and $97 \%$ were within $1^{\circ}$ ). Larger errors in patient position occurred infrequently. They also analyzed the impact of a stricter threshold for patient repositioning. They found that there was a statistically significant difference in precision if a 1 -mm threshold was used instead of a $1.5-\mathrm{mm}$ threshold (intrafraction translational motion was $0.5 \pm 0.4$ and $0.7 \pm 0.5$, respectively). This suggested that positioning the patient as precisely as possible reduces subsequent out-of-tolerance motion and improves the overall precision of treatment delivery.

For cervical and upper thoracic spinal lesions above T4, we utilize a five-point thermoplastic head mask similar to several other institutions [9]. If a unit that allows for real-time intrafraction image guidance such as CyberKnife is used, then a regular vacuum cushion or alpha cradle is sufficient [11].

Imaging for treatment delivery

IGRT has allowed for the delivery of complicated SBRT treatment plans with a high degree of accuracy. The patient is imaged while he or she is immobilized on the treatment table, thereby providing the practitioner with the opportunity to match the pretreatment position of the tumor to that at the time of simulation and determining the positioning changes necessary prior to treatment delivery. The IGRT systems can be broken down into those based on stereoscopic X-ray and CTbased imaging systems. Systems such as CyberKnife and Novalis BrainLab (BrainLab AG and Varian Medical Systems) utilize stereoscopic $\mathrm{x}$-ray systems for intrafraction imaging. Orthogonal x-rays are processed by software to generate indirect $3 \mathrm{D}$ information regarding the target position. The difference between CyberKnife and Novalis BrainLab lies in the fact that the linear accelerator is mounted on a robotic arm on the CyberKnife system, which can adjust automatically to small changes in patient movement whereas in Novalis BrainLab, the treatment halts until positional changes are manually performed. A kilovoltage-based CT image guidance system mounted onto a linear accelerator has been named CBCT. CBCT offers an important advantage over x-ray-based systems as CBCT imaging results in acquisition of high-quality volumetric imaging of not only the body structures but also soft tissues including the spinal cord and tumor. Although CBCT provides volumetric imaging, its main disadvantage is the time necessary to perform CBCT. The interested reader is referred to a recent review by Dahele et al. which discusses the clinical applications of imaging in SSBRT [12].

Treatment planning

\section{Target volume delineation}

The gross tumor volume (GTV) is defined as the radiographically visible tumor based on contrast-enhanced MRI. The clinical target volume (CTV) is defined as the margin applied to the GTV to account for potential microscopic disease in the vicinity of GTV. A margin around the CTV to account for daily patient setup errors is called the planning target volume (PTV). There is considerable variation between centers regarding the volume to which radiation dose is prescribed, and no consensus regarding a standard currently exists. In general, most centers conform to one of two methodologies for delineating the target volume. UCSF, Pittsburgh, and Stanford utilize CT imaging to contour the GTV without any additional margin added to account for microscopic disease similar to target delineation used in radiosurgery for brain metastases (i.e., $\mathrm{CTV}=\mathrm{GTV}$ ) [13-15]. The margins for PTV have ranged from 0 to $10 \mathrm{~mm}$ with adjustments for neural contours [4]. Practitioners at Henry Ford Hospital and MDACC utilize MRI to contour the GTV with additional CTV margins added based on anatomic routes of spread within the vertebral segment [16, 17]. A recent study analyzing the differences among five institutions regarding their practice of sSBRT demonstrated that the details of target volume definition are quite different [9]. Table 1 demonstrates the differences between centers and the definition we use at the Cleveland Clinic, and also shows the definition used by the Radiation Therapy Oncology Group (RTOG) phase II/III sSBRT trial (RTOG 0631). At the Cleveland Clinic, we define the target according to the current RTOG study which defines a CTV incorporating the tumor. This CTV is location based to incorporate areas of potential local spread. We do not add a PTV margin.

\section{Spinal cord contouring and dose limits}

The most critical OAR in sSBRT is the spinal cord as radiation myelopathy may result in paralysis. Therefore, the spinal cord is the strict dose-limiting structure. 
Table 1 Differences between centers and the definition used by the Radiation Therapy Oncology Group (RTOG) phase II/III sSBRT trial (RTOG 0631)

\begin{tabular}{|c|c|c|c|}
\hline Institution & $\begin{array}{l}\text { Imaging } \\
\text { modality }\end{array}$ & Treatment unit & Planning target volume definition \\
\hline Henry Ford Hospital [16] & $\begin{array}{l}\mathrm{CT} / \mathrm{MRI} \\
\text { fusion }\end{array}$ & Novalis (BrainLab) & $\begin{array}{l}\text { Entire involved spinal segment }+ \text { gross } \\
\text { epidural/paraspinal disease }\end{array}$ \\
\hline University of Heidelberg, Germany [38] & $\begin{array}{l}\text { CT/MRI } \\
\text { fusion }\end{array}$ & 6/15-MV linear accelerator (Siemens) & GTV+entire vertebral body \\
\hline University of Florida [21] & $\begin{array}{l}\mathrm{CT} / \mathrm{MRI} \\
\text { fusion }\end{array}$ & Synergy-S (Elekta) & $\begin{array}{l}\text { GTV }+10-\mathrm{mm} \text { bone margin } \pm 2-\mathrm{mm} \text { extension beyond } \\
\text { the bone cortex if GTV is close to the bone surface. } \\
\text { No margins added to epidural disease GTV }\end{array}$ \\
\hline RTOG 0631 [39] & $\begin{array}{l}\text { CT/MRI } \\
\text { fusion }\end{array}$ & Various & $\begin{array}{l}\text { GTV } \pm \text { vertebral body } \pm \text { right and left pedicles } \\
\text { (depending on GTV location) }\end{array}$ \\
\hline Cleveland Clinic [23] & $\begin{array}{l}\mathrm{CT} / \mathrm{MRI} \\
\text { fusion }\end{array}$ & Novalis (BrainLab) & $\begin{array}{l}\text { GTV } \pm \text { vertebral body } \pm \text { right and left pedicles } \\
\text { (depending on GTV location) }\end{array}$ \\
\hline MDACC [17] & $\mathrm{CT}$ & EXaCT Targeting System (Varian) & $\begin{array}{l}\text { GTV +entire vertebral body }+ \text { potential areas } \\
\text { of spinal extension }\end{array}$ \\
\hline MSKCC [40] & $\mathrm{CT}$ & EXaCT Targeting System (Varian) & GTV $+10-m m$ expansion except at the cord \\
\hline UPMC [14] & $\mathrm{CT}$ & CyberKnife & GTV \\
\hline Stanford [15] & $\mathrm{CT}$ & CyberKnife & Target lesion $+2-\mathrm{mm}$ margin \\
\hline UCSF [13] & $\mathrm{CT}$ & CyberKnife & GTV \\
\hline
\end{tabular}

Reirradiation using SBRT is challenging because of the paucity of data regarding the tolerance of the spinal cord. Other OARs in SSBRT to consider include the esophagus, kidneys, and bowel in select patients. The following discussion will focus on differences in spinal cord contouring and spinal cord dose limits between institutions.

Institutional practices regarding contouring of neural critical structures (NCS), which include the spinal cord and cauda equina, are varied among institutions (Table 2). Some examples of delineating the NCS include contouring the spinal cord ( \pm margins for setup errors), the spinal canal, and the thecal sac. At MDACC, the intramedullary spinal cord (and thecal sac for cauda equina) is contoured with no applied margin based on CT imaging post intrathecal administration of iohexol [17]. However, they do add a 2-mm expansion to account for setup and contouring uncertainty. At UCSF, they contour the spinal canal or thecal sac based on CT imaging with $20-\mathrm{mm}$ cranial and caudal margins to account for positional uncertainties [13]. This serves to provide some margins to the actual spinal cord given that radiation delivery in CyberKnife is not coplanar. Intrafraction patient and organ motion may also affect the estimated dose to NCS. This is illustrated by Cai's study which utilized dynamic MRI to show that intrafraction patient movement in the thoracic spinal cord was limited to $<0.5 \mathrm{~mm}$ [18]. Furthermore, Ma et al. showed that intrafraction motion was greatest in the cervical spinal cord compared to the thoracolumbar cord and that frequent intrafraction imaging was necessary to ensure accurate delivery of the radiation dose [11]. It is also important to note that when MRI and CT fusion are utilized for contouring NCS, it is important to account for fusion uncertainties when determining the margins that need to be added. Guckenberger et al. studied the practices of five institutions and report that four of the institutions utilized MRI for contouring the spinal cord while one institution utilized CT to contour the spinal canal [9]. All five institutions contoured the NCS one vertebral body above and below the PTV and 1to 2-mm safety margins are applied.

Similar to the differences in NCS contouring, institutional differences in spinal cord and cauda equina dose limits used for treatment planning are extensive and summarized in Table 2. At the Cleveland Clinic, we contour the spinal cord based on MRI-CT fusion and add 6-mm cranial and caudal margins to the spinal cord to account for dose fall off superior and inferior to the region treated. For the cauda equina, we contour the thecal sac with $6-\mathrm{mm}$ cranial and caudal margins as well. We limit the spinal cord to a maximum dose of $<14$ Gy and limit $10 \%$ of the cord to $10 \mathrm{~Gy}$ or more. For the cauda equina, we limit the maximum dose to $<16$ Gy and limit $10 \%$ of the cauda to 12 Gy or more (Figs. 3, 4, and 5).

\section{Treatment dose and fractionation}

Similar to the differences in the definition of a target volume between institutions, there is no consensus regarding radiation dose or fractionation scheme. Currently, most centers use either a single-fraction approach or a hypofractionated regimen. Single-fraction doses tend to range from 12 to $24 \mathrm{~Gy}$. Hypofractionated regimens consist mainly of $25 \mathrm{~Gy}$ in five fractions, $30 \mathrm{~Gy}$ in five fractions, $24 \mathrm{~Gy}$ in three fractions, $24 \mathrm{~Gy}$ in two fractions, and $27 \mathrm{~Gy}$ in three fractions. Table 3 demonstrates selected fractionation schemes reported in the literature and their reported outcomes. At the Cleveland Clinic, we have 
Table 2 Institutional practices regarding contouring of neural critical structures

\begin{tabular}{|c|c|c|c|}
\hline Institution & $\begin{array}{l}\text { Imaging } \\
\text { modality }\end{array}$ & NCS contour definition & Threshold criteria for NCS \\
\hline Henry Ford Hospital [16] & $\mathrm{CT} / \mathrm{MRI}$ fusion & $\begin{array}{l}\text { Spinal cord: spinal cord }+6-\mathrm{mm} \\
\text { cranial and caudal extensions }\end{array}$ & $\begin{array}{l}\text { Spinal cord: } 10 \text { Gy or more } \\
\text { to } 10 \% \text { or less of PTV; } \\
\text { cauda equina: } 12 \text { Gy or } \\
\text { more to } 10 \% \text { or less of PTV }\end{array}$ \\
\hline University of Heidelberg, Germany [38] & $\mathrm{CT} / \mathrm{MRI}$ fusion & $\begin{array}{l}\text { Spinal cord: spinal cord }+2-3 \\
\text { safety margin }\end{array}$ & $\begin{array}{l}\text { Reirradiation: }<20 \mathrm{~Gy} / 10 \mathrm{fx} / \text { median } \% \\
\text { of cord }>30 \% \text { prescribed } \\
\text { dose: } 23-40.5 \%\end{array}$ \\
\hline University of Florida [21] & $\mathrm{CT} / \mathrm{MRI}$ fusion & $\begin{array}{l}\text { Spinal cord or cauda equina } \\
\text { with margin of } 1 \text { spinal level } \\
\text { above and below }\end{array}$ & $\begin{array}{l}\text { No history of RT: } 12 \text { Gy to } 1 \mathrm{cc} \text {; } \\
\text { history of prior RT: } 5 \text { Gy to } 5 \mathrm{cc}\end{array}$ \\
\hline RTOG [39] & $\mathrm{CT} / \mathrm{MRI}$ fusion & $\begin{array}{l}\text { Spinal cord: spinal cord }+6-\mathrm{mm} \\
\text { cranial and caudal extensions }\end{array}$ & $\begin{array}{l}\text { Spinal cord: }<10 \mathrm{~Gy} \text { to } 10 \% \mathrm{PTV} \\
\text { and limit } 0.35 \mathrm{cc} \text { to }<10 \mathrm{~Gy} \\
\text { and limit } 0.03 \mathrm{cc} \text { to }<14 \mathrm{~Gy} ; \\
\text { cauda equina: limit }<0.03 \mathrm{cc} \\
\text { to } 16 \mathrm{~Gy} \text { and limit }<5 \mathrm{cc} \text { to } 14 \mathrm{~Gy}\end{array}$ \\
\hline $\mathrm{CCF}$ & $\mathrm{CT} / \mathrm{MRI}$ fusion & $\begin{array}{l}\text { Spinal cord: spinal cord }+6-\mathrm{mm} \\
\text { cranial and caudal extensions }\end{array}$ & $\begin{array}{l}\text { Spinal cord: } 10 \text { Gy or more } \\
\text { to } 10 \% \text { or less of PTV and } \\
\text { max dose }<14 \text { Gy; cauda } \\
\text { equina: } 12 \text { Gy or more to } \\
10 \% \text { or less and max dose }<16 \text { Gy }\end{array}$ \\
\hline MDACC [41] & CT (with intrathecal iohexol) & $\begin{array}{l}\text { Spinal cord: spinal cord; } \\
\text { cauda equina: thecal sac; } \\
\text { no applied margin }\end{array}$ & Max dose $=10 \mathrm{~Gy}$ \\
\hline MSKCC [27] & MRI or CT myelogram & $\begin{array}{l}\text { Spinal cord: spinal cord; } \\
\text { cauda equina: thecal sac }\end{array}$ & $\begin{array}{l}\text { Spinal cord max dose }=14 \mathrm{~Gy} \\
\text { cauda equina } \max \text { dose }=16 \mathrm{~Gy}\end{array}$ \\
\hline UPMC [9] & MRI & $\begin{array}{l}\text { Spinal cord: spinal cord; } \\
\text { cauda equina: thecal sac; } \\
\text { margin of } 1 \text { spinal level } \\
\text { above and below }\end{array}$ & $\begin{array}{l}\text { Spinal cord: } \max \text { dose }=11 \text { Gy }(1 \mathrm{fx}) \\
\text { or } 18 \mathrm{~Gy}(3 \mathrm{fx}) \text {; cauda equina: } \\
12 \text { Gy }(1 \mathrm{fx}) \text { or } 18 \text { Gy }(3 \mathrm{fx})\end{array}$ \\
\hline Stanford [15] & $\mathrm{CT}$ & Spinal cord: spinal cord & Max dose $=10$ Gy in single fraction \\
\hline UCSF [13] & $\mathrm{CT}$ & $\begin{array}{l}\text { Spinal cord: thecal sac; } \\
\text { cauda equina: thecal } \\
\text { sac; } 20 \text {-mm margins }\end{array}$ & Not published \\
\hline
\end{tabular}

escalated our standard single-fraction dose since the inception of our program from 12 to 18 Gy currently. Given that BED calculations may be inaccurate in the setting of SBRT, comparing different regimens in a scientific manner has been difficult [19]. As a result, accurate mathematical models that are applicable to SBRT are needed in order to compare different fractionation schemes and develop a consensus regarding the optimal treatment strategy for spinal tumors and metastases.

\section{Clinical applications}

\section{Indications for spine SBRT}

Spine SBRT is typically performed in a single or limited number of fractions and offers the potential for more durable pain control as well as long-term local tumor control. Patients with a long life expectancy, high Karnofsky Performance Score (KPS), resistant histology, limited spinal metastases, and oligometastatic disease are generally considered good candidates for spine SBRT. The patient must also be able to tolerate the treatment, and if they have received prior CRT, a total cord dose of $<45$ Gy from prior CRT is thought to be ideal. Given the accuracy of most SBRT delivery systems, a separation of at least 3-5 $\mathrm{mm}$ between tumor and cord is desirable [4, 6, 20].

Very short life expectancy, low KPS and significant cord compression, mechanical instability of the spine, history of a connective tissue disorder, and prior radiotherapy within the last 3 months are relative contraindications for spine SBRT. Some centers exclude patients that have radiosensitive histologies [9], but at our center, we have offered spine SBRT to select patients with radiosensitive histologies, typically with oligometastatic disease.

Local control and predictors of local control and overall survival

The majority of published studies of spine SBRT are retrospective reviews. Very limited numbers of prospective trials 


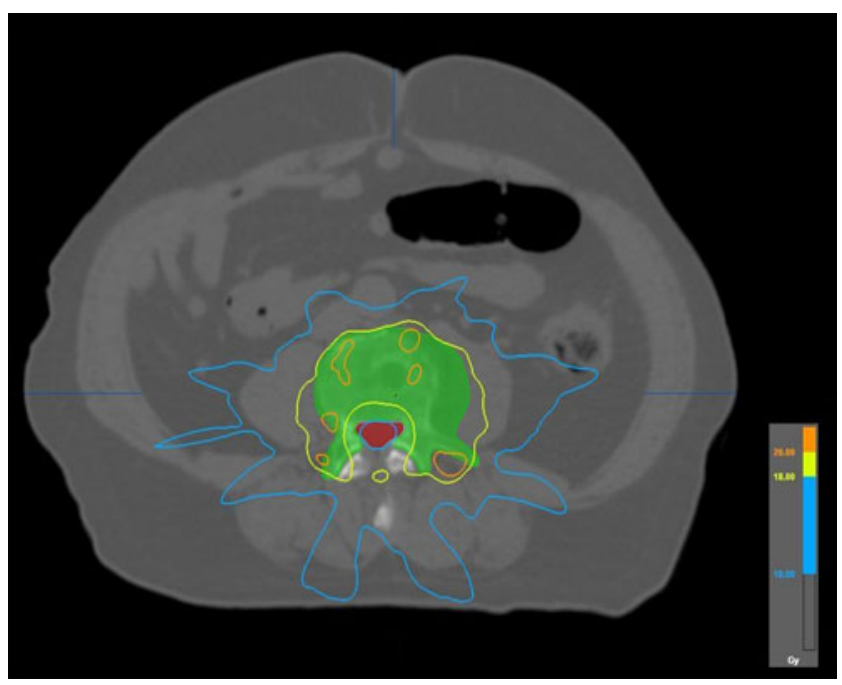

Fig. 3 Vertebral metastasis (L4) from RCC treated with SBRT. The prescription dose to the PTV (green) is 18 Gy (yellow IDL). The 10 Gy IDL in blue is seen sparing the thecal sac. A 14-field IMRT/IGRT was used to deliver a total dose of 18 Gy to the PTV

have been performed. Several articles provide an in-depth review of the spine SBRT literature [3, 4, 6]; here, we provide a summary of select studies.

Chang et al. reported the results of their phase I/II study which included 63 patients with 74 spinal metastases treated with SBRT to a dose of either $30 \mathrm{~Gy}$ in five fractions or $27 \mathrm{~Gy}$ in three fractions. Seventeen patients developed tumor progression and 37 died. The 1-year actuarial radiographic progression-free survival was $84 \%$ with a median follow-up of 21.3 months [17]. Amdur et al. reported the results of their phase II trial which included 21 patients with 25 spinal metastases who were treated with SBRT to a dose of $15 \mathrm{~Gy}$ in one fraction. Local control rate was $95 \%$ with $43 \%$ of patients reporting improvement in pain. One-year overall progressionfree survival was $5 \%$ secondary to most patients developing progressive systemic disease [21]. Multiple retrospective studies of SBRT for spinal metastases have demonstrated local control rates ranging from 80 to $100 \%$. However, important differences exist in the criteria for local control; some studies use pain relief, some use radiographic control, some use clinical control, while others use combined metrics. Although varied endpoints make comparisons difficult, both retrospective and prospective studies have shown spine SBRT to be efficacious at controlling local tumor growth as well as providing pain relief (Table 3 ).

Several studies have shown that typical patient and tumor factors such as sex, age, KPS, systemic burden of disease, target volume, and various tumor dosimetric data have failed to predict local control. Sahgal et al. suggest that distance separating target volume from spinal cord may be predictive of local control [13]. Choi et al. further suggest that time interval greater than 12 months for retreatment is predictive of superior local control [22]. Chao et al. recently performed a recursive partitioning analysis (RPA) for patients undergoing spinal SBRT [23]. RPA was performed to identify associations between overall survival and a variety of variables including histology, gender, age, KPS, control of primary disease, extraosseous metastases, time from primary diagnosis, SBRT dose ( $\leq 14$ vs. $>14$ Gy), extent of spine disease, up front or salvage SBRT, presence of paraspinal extension, and previous surgical intervention. $\mathrm{He}$ found that overall survival was predominantly associated with global patient and diseases characteristics. Patients with a KPS $>70$ and time from primary diagnosis (TPD) $>30$ months (class 1 ) had the longest median overall survival of 21.1 months $(n=59)$. Class 2 patients were those that had a KPS $\leq 70$ and TPD $>30$ months or age $<70$ years and TPD $\leq 30$ months, and they had a median overall survival of 8.7 months $(n=104)$. Class 3 patients had the lowest median overall survival ( 2.4 months; $n=11$ ) and were $\geq 70$ years old and had TPD $\leq 30$ months. Interestingly, this classification also helps identify those patients that have a short life expectancy and thus are better candidates for conventional radiotherapy $[9,20]$ (Table 4$)$.

Currently, the first phase III trial, RTOG 0631, tests whether SBRT (single dose of 16 to $18 \mathrm{~Gy}$ ) improves pain control as compared to conventional external beam radiotherapy (single dose of $8 \mathrm{~Gy}$ ) and is accruing patients. However, results from this study are not expected to be available in the near future.

Impact of dose on local control

A review of the literature does not reveal a consistent sSBRT dose-fractionation scheme for the treatment of spinal metastases (Table 3); some centers prefer a multifraction

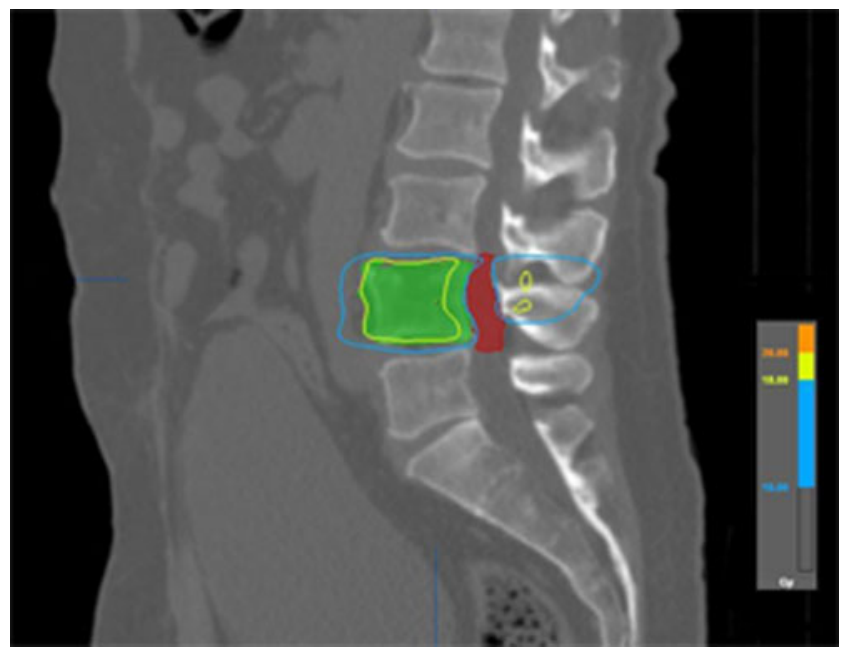

Fig. 4 Coronal view showing PTV in green (L4 vertebral body) and thecal sac in red. Again, the 10 Gy IDL (in blue) is seen sparing the OAR. A 14-field IMRT/IGRT was used to deliver a total dose of 18 Gy (IDL in yellow-green) to the PTV 


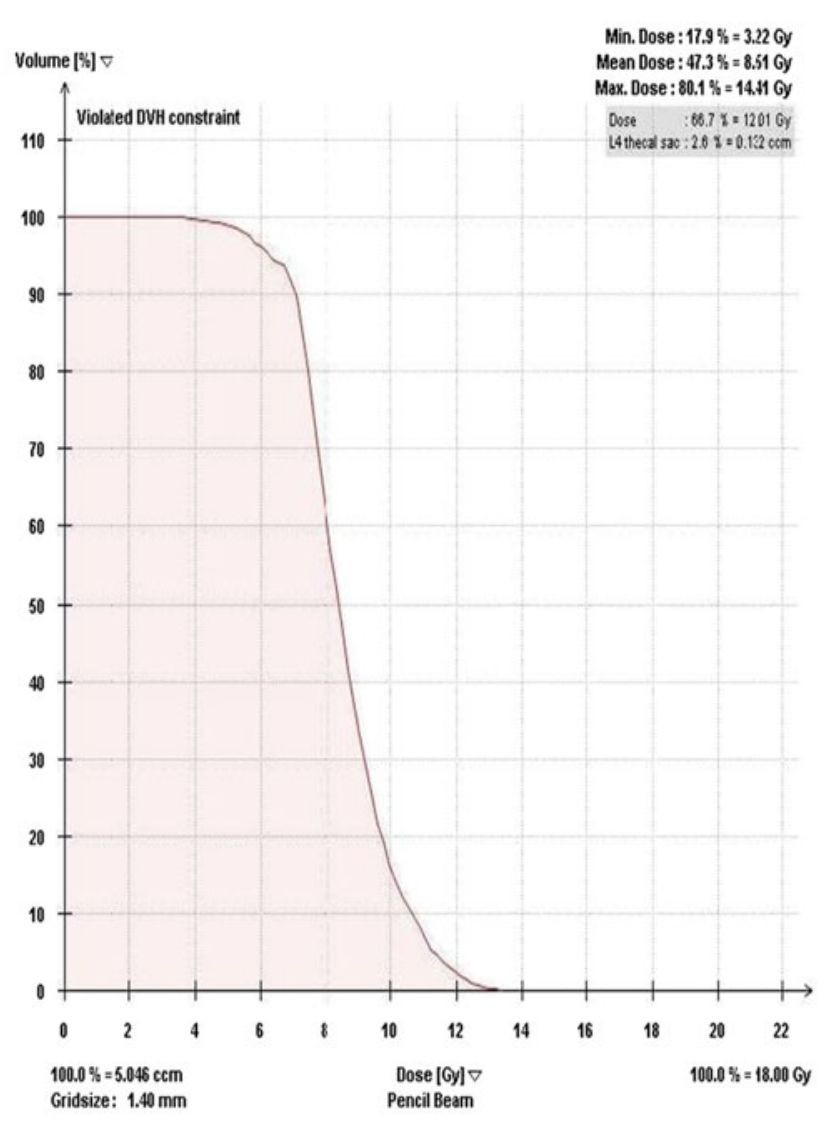

Fig. 5 Pink dose-volume histogram (DVH) showing the OAR dose parameters and the green DVH showing the same for the PTV. The maximum point dose delivered to the thecal sac using a 14-field IMRT/

dosing regimen whereas other centers prefer a singlefraction regimen. Several studies have shown that higher SBRT doses lead to better local control irrespective of tumor histology [19]. Yamada and colleagues at MSKCC examined 93 consecutive patients with 103 spinal metastases treated with sSBRT to doses of 18-24 Gy (median, 24 Gy). They found that while tumor histology was not a statistically significant predictor of local control, a higher radiation dose was associated with improved local control $(p=0.03)$ [24]. In a separate analysis, the MSKCC group studied whether local control was dependent on dose insufficiency. They included 91 consecutively treated lesions in 79 patients and studied the correlation between $D(\min ), D$ (98\%), and $D(95 \%)$ of the GTV and local failure. They found that the dosimetric distributions of treatments that resulted in local failure were statistically different from the corresponding distributions of the entire patient population included in the study. Furthermore, they found that no local failures resulted when $D(\min )$ was $>15$ Gy [25]. Garg et al. studied 59 patients with 63 spinal tumors and found that of the tumors that progressed post-sSBRT, $81 \%$ had an epidural component with $5 \mathrm{~mm}$ of the spinal cord and many of

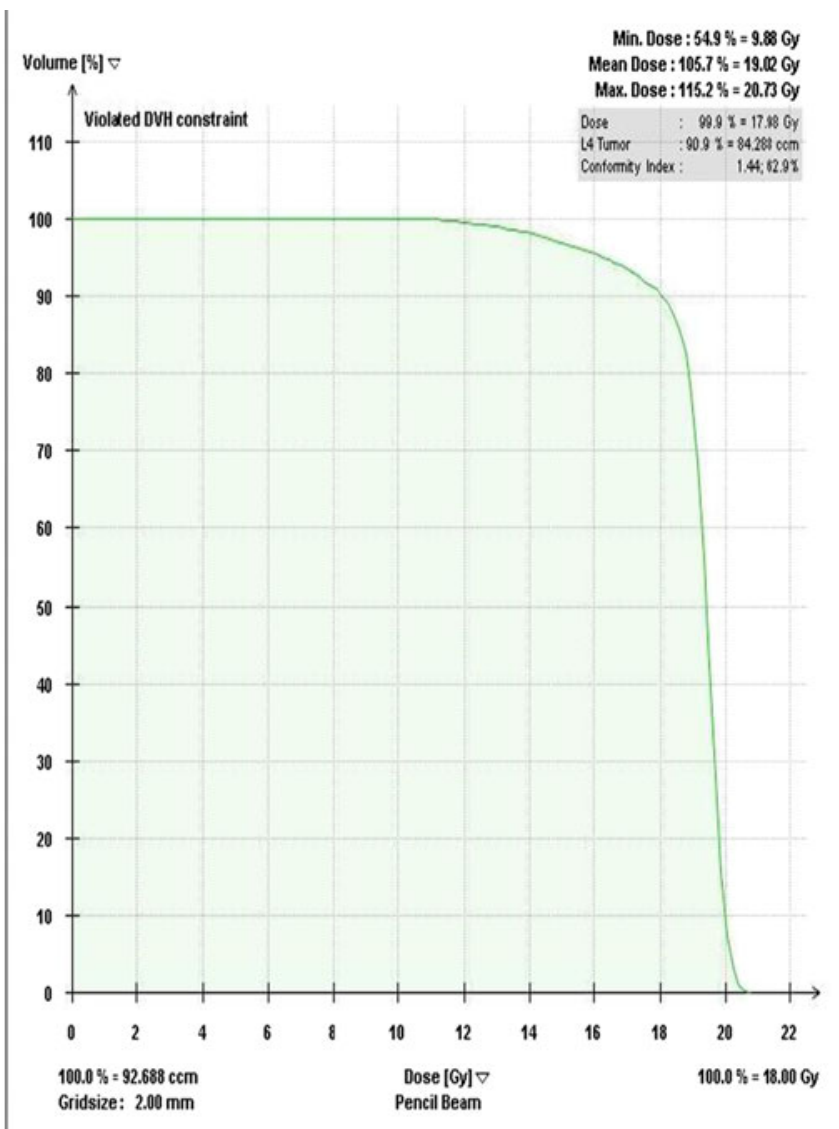

IGRT is $14.41 \mathrm{~Gy}$ (dose constraint, 16 Gy). The mean dose to the PTV is $19.02 \mathrm{~Gy}$, and the prescription dose is $18 \mathrm{~Gy}$

them eventually developed spinal cord compression [26]. This suggests that dose insufficiency in the epidural space (due to sparing of the spinal cord to prevent radiation myelopathy) leads to local progression. Damast et al. studied patients who were treated with sSBRT after in-field recurrence using a dose of either $4 \mathrm{~Gy} \times 5$ fractions $(n=42)$ or $6 \mathrm{~Gy} \times 5$ fractions $(n=55)$. They found that the group treated with the higher dose had a lower rate of local failure than the lower-dose group ( 26 vs. $45 \%, p=0.04$, respectively) [27]. Important to note, however, is that the lowerdose group in Damast's study was treated with a dose scheme commonly used in conventional radiotherapy. Choi et al. reviewed their experience with sSBRT in the retreatment setting after in-field recurrence. They reviewed 42 patients with 51 lesions who were treated at a median dose of $20 \mathrm{~Gy}$ (range, 10-30) in one to five fractions (median, 2). To compare differing dosing schemes, they used the linear quadratic model $(\alpha / \beta=3$ for the spinal cord and $\alpha / \beta=10$ for the tumor) to calculate the maximum single-session equivalent dose (SSED). Their analysis showed that tumor recurrence within 12 months of initial radiotherapy and SSED $<15 \mathrm{~Gy}_{10}$ were significant predictors of local failure [22]. 
Table 3 Selected fractionation schemes reported in the literature and their reported outcomes

\begin{tabular}{|c|c|c|c|c|c|c|}
\hline Study & Type of study & $\begin{array}{l}\text { No. of pts/no. } \\
\text { of tumors }\end{array}$ & Indication & Prescription dose & $\begin{array}{l}\text { Media } \\
\text { follow-up }\end{array}$ & Outcomes \\
\hline Chang et al. [17] & Phase I/II & $63 / 74$ & Mixed & $\begin{array}{c}30 \mathrm{~Gy} / 5 \mathrm{fx} \text { or } \\
27 \mathrm{~Gy} / 3 \mathrm{fx}\end{array}$ & 21.3 months & 1 year PFS, $84 \%$ \\
\hline Amdur et al. [21] & Phase I/II & $21 / 25$ & Mixed & $\begin{array}{l}24 \mathrm{~Gy} / 3 \mathrm{fx} \text { or } \\
25-30 \mathrm{~Gy} / 5 \mathrm{fx} \\
\text { (if PTV touched cord) }\end{array}$ & 8 months & $\mathrm{LC}=95 \%$, pain relief $=43 \%$ \\
\hline Gerszten et al. [14] & Retrospective & $393 / 500$ & Mixed & $\begin{array}{l}20 \mathrm{~Gy} / 1 \mathrm{fx} \text { (range, } \\
12.5-25 \mathrm{~Gy})\end{array}$ & 21 months & $\begin{array}{l}\mathrm{LC}=88 \% \\
\text { pain relief }=86 \% ; \\
\text { neurological } \\
\text { improvement }=85 \%\end{array}$ \\
\hline Gibbs et al. [15] & Retrospective & $74 / 102$ & Mixed & $14-25 \mathrm{~Gy} / 1-5 \mathrm{fx}$ & 9 months & Pain relief $=84 \%$ \\
\hline Yamada et al. [24] & Retrospective & $93 / 103$ & Mixed & $\begin{array}{l}24 \mathrm{~Gy} / 1 \mathrm{fx} \\
\text { (range, 18-24) }\end{array}$ & 15.7 months & Actuarial LC $=90 \%$; \\
\hline Mahadevan et al. [42] & Retrospective & $60 / 81$ & Reirradiated & $\begin{array}{l}24 \mathrm{~Gy} / 3 \mathrm{fx} \text { or } \\
25-30 \mathrm{~Gy} / 5 \mathrm{fx} \\
\text { (if PTV touched cord) }\end{array}$ & 12 months & $\begin{array}{l}\text { R-LC } 93 \% \text {, } \\
65 \% \text { pain control }\end{array}$ \\
\hline Damast et al. [27] & Retrospective & $97 / 97$ & Reirradiated & 20 or $30 \mathrm{~Gy} / 5 \mathrm{fx}$ & 12.1 months & $\begin{array}{c}\text { LC, } 20 \text { Gy }=55 \% \\
30 \mathrm{~Gy}=74 \%\end{array}$ \\
\hline Ryu et al. [43] & Retrospective & $62 / 85$ & $\begin{array}{l}\text { Spinal cord } \\
\text { compression }\end{array}$ & $\begin{array}{l}24 \mathrm{~Gy} / 1 \mathrm{fx} \\
\text { (range, 12-20) }\end{array}$ & 10.3 months & $\begin{array}{l}\text { Tumor response rate }=80 \% \\
65 \% \text { reduction in tumor } \\
\text { volume at } 2 \text { months; } \\
63 \% \text { of those with neurologic } \\
\text { deficits showed improvement }\end{array}$ \\
\hline
\end{tabular}

Some other studies have failed to provide significant evidence that a dose response with sSBRT exists. Colleagues at MDACC observed that the local control did not differ between those patients that were treated with 30 Gy in five fractions vs. those patients treated with $27 \mathrm{~Gy}$ in three fractions. In one of the first papers showing that sSBRT is safe and effective in patients with prior history of radiation, Sahgal et al. studied 39 consecutive patients with 60 metastases. The median dose prescribed was 24 Gy in three fractions prescribed to the 67 and $60 \%$ isodose for the unirradiated and reirradiated patients, respectively. There was no significant difference in progression-free survival between reirradiated patients vs. all other patients $(p=0.31)$ [13].

No consensus exists regarding which dosing scheme is superior: single fraction or multifraction. Single-fraction SBRT is ideal for small target volumes. However, because of the large dose spill associated with large target volumes, the use of fractionated SSBRT may be considered to avoid excessive dose to the spinal cord by improving the therapeutic ratio. Furthermore, fractionated SBRT offers traditional radiobiological advantages: reoxygenation, reassortment, and repair [19]. However, Kim et al. recently showed in a rat model that fractionation of SBRT leads to decreased tumor kill efficiency [28]. Furthermore, Qutob et al. showed in an in vitro study that cells that received prior fractionated radiation had increased radioresistance in comparison to cells that were radiation-naive independent of intrinsic radiosensitivity of the cells [29]. These studies show that although fractionated radiotherapy offers some advantages in the treatment of large tumors, single-fraction SBRT is likely more effective at achieving superior local control than fractionated SBRT. However, these are preclinical data, and thus far, clinical data showing superiority of singlefraction SBRT to multifraction SBRT do not exist. Further work needs to be done to determine which fractionation scheme is optimal for treating spinal metastases using SBRT.

\section{Patterns of failure}

In contrast with conventional radiotherapy, sSBRT is highly focused to the target, and most centers do not add margins to the PTV. The epidural space has been identified as an area at an elevated risk for failure. Multiple series have identified that the closer the proximity of epidural disease to the spinal cord, the higher the risk for failure in the epidural space $[13,22,26]$. It is thought that this could be related to relative underdosing of the target volume in order to spare the spinal cord, due to

Table 4 Recursive partitioning analysis for patients undergoing spinal SBRT [23]

\begin{tabular}{lll}
\hline RPA class & Criteria & Overall survival \\
\hline I & TPD $>30$ months and KPS $>70$ & 21 months \\
II & TPD $>30$ months and KPS $\leq 70$ & 8.7 months \\
& TPD $\leq 30$ months and age $<70$ y & \\
II & TPD $\leq 30$ months and age $\geq 70$ y & 2.4 months \\
\hline
\end{tabular}


microscopic epidural disease that does not receive the full radiation dose due to lack of margins in the epidural space, or inevitably due to aggressive tumor biology [3].

The risk of failure in the adjacent vertebral body is generally thought to be low [30]. Recently, Koyfman et al. showed that in their experience, failure in the adjacent vertebral body was $12.5 \%$ and was associated with the presence of paraspinal disease and dose $<16$ Gy [31]. The results of this study suggest that perhaps microscopic disease in the paraspinal area led to failure in the adjacent vertebral body. It is also conceivable that microscopic disease in the epidural space may also be partially responsible for epidural failure. For a more detailed discussion of patterns of failure, we refer the reader to recent a critical review by Sahgal et al. [30].

\section{Toxicities}

The most common acute toxicities from sSBRT are grade 1-2 fatigue (up to $40 \%$ ) and gastrointestinal effects (up to 10-20\%) [3]. These side effects usually do not result in long-term consequences for patients. However, the most feared toxicity of sSBRT is radiation myelopathy, which is rarely reported with conventional radiotherapy. Development of radiation myelopathy rarely occurs within 6 months of treatment and almost always presents within 3 years of treatment [32]. The incidence of radiation myelopathy from sSBRT has been estimated to be $<1 \%$ [33]. Recently, Sahgal et al. performed a multi-institutional study of five cases of radiation myelopathy who had not received prior radiotherapy for spinal metastases and compared it to 19 patients with no radiation myelopathy post-sSBRT [34]. Out of the five patients that developed myelopathy, three patients received a maximum point dose of 10.6, 13.1, and $14.8 \mathrm{~Gy}$ in one fraction to the thecal sac. The other two patients received 25.6 Gy in two fractions and 30.9 Gy in three fractions to the thecal sac. His analysis showed that a thecal sac maximum point dose of up to $10 \mathrm{~Gy}$ in one fraction is safe. Sahgal et al. also modeled his data using BED and determined that 30-35 2 Gy equivalent BED for up to five fractions was a safe dose range. It is important to note that Sahgal et al. studied the dose to the entire thecal sac rather than the true spinal cord. Radiation myelopathy has also been observed in patients who underwent sSBRT after initial conventional radiotherapy (prior dose ranging from $25.2 \mathrm{~Gy}$ in 28 fractions to $51.9 \mathrm{~Gy}$ in 28 fractions). sSBRT doses used for these patients were 14 or 16 Gy in one fraction, 20 or 21 Gy in two fractions, or $33 \mathrm{~Gy}$ in three fractions [35].

Spinal cord tolerance in the reirradiation setting is also an active area of research, and centers lower the prescribed dose in this setting to decrease the risk of radiation myelopathy. Damast et al. from MSKCC demonstrate that when the dose of sSBRT was increased from 4 to $6 \mathrm{~Gy} \times 5$ fractions in the reirradiation setting, the local failure rate decreased from 45 to $26 \%$ without increasing the risk of radiation myelopathy (median follow-up=12.1 months) [27]. The major critique of Damast et al.'s study is the short follow-up, and therefore, it is conceivable that many patients did not survive long enough to develop toxicity. Long-term follow-up data are insufficient to calculate a dose-volume relationship especially because of the short survival of patients with spine metastases [33]. Aggregating all the available clinical data on spinal cord myelopathy, Kirkpatrick et al. concluded that $13 \mathrm{~Gy}$ in one fraction or $20 \mathrm{~Gy}$ in three fractions confers a risk of myelopathy of less than $1 \%$ each [33]. The risk for radiation myelopathy from repeat $\mathrm{SSBRT}$ after initial SSBRT is also not known.

Vertebral body fracture is also a significant toxicity of sSBRT and can lead to significant morbidity in patients. Colleagues from MSKCC studied 62 consecutive patients with 71 vertebral bodies treated to a range of doses from 18 to 24 Gy. They reported that $39 \%$ of patients treated with high-dose single-fraction image-guided radiotherapy for spinal metastases developed new or progressive vertebral fractures [36]. Their analysis of risk factors suggested that the following were risk factors for vertebral fractures post-sSBRT: location between T10 and sacrum, lytic appearance, and $>40 \%$ vertebral involvement. Furthermore, patients who developed fractures had higher narcotic usage, worse KPS, and higher pain scores [36]. More recently, colleagues from MDACC reported their experience. Boehling et al. studied 123 vertebral bodies in 93 patients with sSBRT dose of one, three, or five fractions for overall median doses of 18,27 , and $30 \mathrm{~Gy}$, respectively. They report a fracture rate of $20 \%$ with age $>55$ years, preexisting vertebral fractures, and baseline pain as significant risk factors associated with fracture progression [37]. In the Cleveland Clinic series of 57 patients (88 treated vertebral bodies) treated for spinal metastases from renal cell carcinoma with a median dose of 15 Gy in one fraction, we report a $14 \%$ risk of new or progressive vertebral fractures (Balagamwala et al., submitted). Identifying patients at risk for developing vertebral fractures is important as these patients may benefit from prophylactic kyphoplasty or vertebroplasty. Moving forward, it will be important to determine whether sSBRT dose is related to the development of vertebral fractures as dose de-escalation may not necessarily compromise local control but may prevent patients from undergoing additional medical and surgical procedures that may not be otherwise required.

\section{Conclusion and future directions}

Spinal SBRT is an emerging radiotherapy technique that could change current clinical practice. Early data from numerous prospective and retrospective case series suggest 
that sSBRT is safe and effective. Often, patients considered eligible for sSBRT do not have a surgical option due to extensive metastatic disease burden, poor KPS, or coexisting medical comorbidities. Hence, sSBRT becomes a default option for these patients. Currently, sSBRT remains the only effective nonsurgical treatment option for previously irradiated spinal metastasis. The infrequent outpatient treatment visits and the relative lack of acute side effects together with rapid and durable pain relief make sSBRT an attractive treatment option for this patient population. The decreasing opiate dependence and alleviation of opiate side effects should also be recognized as an added benefit of sSBRT.

Currently, the role of sSBRT post laminectomy and decompressive surgery as primary treatment for malignant spinal cord compression instead of conventional EBRT with corpectomy is being explored at the Cleveland Clinic and other institutions. If patients are well selected, organ-at-risk dose constraints are met, and the PTV encompasses all of the local metastatic disease, the risk of myelopathy, vertebral compression fractures, and epidural disease relapse can be minimized.

Conflict of interest There are no relevant conflicts of interest.

\section{References}

1. Chang BK, Timmerman RD (2007) Stereotactic body radiation therapy: a comprehensive review. Am J Clin Oncol 30:637-644

2. Finn MA, Vrionis FD, Schmidt MH (2007) Spinal radiosurgery for metastatic disease of the spine. Cancer Control 14:405-411

3. Masucci GL, Yu E, Ma L et al (2011) Stereotactic body radiotherapy is an effective treatment in reirradiating spinal metastases: current status and practical considerations for safe practice. Expert Rev Anticancer Ther 11:1923-1933

4. Sahgal A, Larson DA, Chang EL (2008) Stereotactic body radiosurgery for spinal metastases: a critical review. Int J Radiat Oncol Biol Phys 71:652-665

5. Chang EL, Shiu AS, Lii M-F et al (2004) Phase I clinical evaluation of near-simultaneous computed tomographic image-guided stereotactic body radiotherapy for spinal metastases. Int J Radiat Oncol Biol Phys 59:1288-1294

6. Lo SS, Sahgal A, Wang JZ et al (2010) Stereotactic body radiation therapy for spinal metastases. Discov Med 9:289-296

7. Hamilton AJ, Lulu BA, Fosmire H, Stea B, Cassady JR (1995) Preliminary clinical experience with linear accelerator-based spinal stereotactic radiosurgery. Neurosurgery 36:311-319

8. Foote M, Letourneau D, Hyde D et al (2011) Technique for stereotactic body radiotherapy for spinal metastases. J Clin Neurosci 18:276-279

9. Guckenberger M, Sweeney RA, Flickinger JC et al (2011) Clinical practice of image-guided spine radiosurgery-results from an international research consortium. Radiat Oncol (London, England) 6:172

10. Hyde D, Lochray F, Korol R et al (2012) Spine stereotactic body radiotherapy utilizing cone-beam $\mathrm{CT}$ image-guidance with a robotic couch: intrafraction motion analysis accounting for all six degrees of freedom. Int J Radiat Oncol Biol Phys 82:e555-e562

11. Ma L, Sahgal A, Hossain S et al (2009) Nonrandom intrafraction target motions and general strategy for correction of spine stereotactic body radiotherapy. Int J Radiat Oncol Biol Phys 75:1261-1265

12. Dahele M, Zindler JD, Sanchez E et al (2011) Imaging for stereotactic spine radiotherapy: clinical considerations. Int $\mathrm{J}$ Radiat Oncol Biol Phys 81:321-330

13. Sahgal A, Ames C, Chou D et al (2009) Stereotactic body radiotherapy is effective salvage therapy for patients with prior radiation of spinal metastases. Int J Radiat Oncol Biol Phys 74:723-731

14. Gerszten PC, Burton SA, Ozhasoglu C, Welch WC (2007) Radiosurgery for spinal metastases: clinical experience in 500 cases from a single institution. Spine 32:193-199

15. Gibbs IC, Kamnerdsupaphon P, Ryu M-R et al (2007) Imageguided robotic radiosurgery for spinal metastases. Radiother Oncol 82:185-190

16. Ryu S, Jin J-Y, Jin R et al (2007) Partial volume tolerance of the spinal cord and complications of single-dose radiosurgery. Cancer 109:628-636

17. Chang EL, Shiu AS, Mendel E et al (2007) Phase I/II study of stereotactic body radiotherapy for spinal metastasis and its pattern of failure. J Neurosurg Spine 7:151-160

18. Cai J, Sheng K, Sheehan JP et al (2007) Evaluation of thoracic spinal cord motion using dynamic MRI. Radiother Oncol 84:279-282

19. Balagamwala EH, Chao ST, Suh JH (2012) Principles of radiobiology of stereotactic radiosurgery and clinical applications in the central nervous system. Technol Cancer Res Treat 11:3-13

20. Lutz S, Berk L, Chang E et al (2011) Palliative radiotherapy for bone metastases: an ASTRO evidence-based guideline. Int $\mathrm{J}$ Radiat Oncol Biol Phys 79:965-976

21. Amdur RJ, Bennett J, Olivier K et al (2009) A prospective, phase II study demonstrating the potential value and limitation of radiosurgery for spine metastases. Am J Clin Oncol 32:515-520

22. Choi CYH, Adler JR, Gibbs IC et al (2010) Stereotactic radiosurgery for treatment of spinal metastases recurring in close proximity to previously irradiated spinal cord. Int J Radiat Oncol Biol Phys 78:499-506

23. Chao ST, Koyfman SA, Woody N et al (2011) Recursive partitioning analysis index is predictive for overall survival in patients undergoing spine stereotactic body radiation therapy for spinal metastases. Int J Radiat Oncol Biol Phys 82:1738-1743

24. Yamada Y, Bilsky MH, Lovelock DM et al (2008) High-dose, single-fraction image-guided intensity-modulated radiotherapy for metastatic spinal lesions. Int J Radiat Oncol Biol Phys 71:484-490

25. Lovelock DM, Zhang Z, Jackson A et al (2010) Correlation of local failure with measures of dose insufficiency in the high-dose single-fraction treatment of bony metastases. Int $\mathrm{J}$ Radiat Oncol Biol Phys 77:1282-1287

26. Garg AK, Wang X-S, Shiu AS et al (2011) Prospective evaluation of spinal reirradiation by using stereotactic body radiation therapy: the University of Texas MD Anderson Cancer Center experience. Cancer 117:3509-3516

27. Damast S, Wright J, Bilsky M et al (2011) Impact of dose on local failure rates after image-guided reirradiation of recurrent paraspinal metastases. Int J Radiat Oncol Biol Phys 81:819-826

28. Kim JH, Khil MS, Kolozsvary A, Gutierrez JA, Brown SL (1999) Fractionated radiosurgery for 9L gliosarcoma in the rat brain. Int $\mathrm{J}$ Radiat Oncol Biol Phys 45:1035-1040

29. Qutob SS, Multani AS, Pathak S et al (2006) Fractionated Xradiation treatment can elicit an inducible-like radioprotective response that is not dependent on the intrinsic cellular X-radiation resistance/sensitivity. Radiat Res 166:590-599 
30. Sahgal A, Bilsky M, Chang EL et al (2011) Stereotactic body radiotherapy for spinal metastases: current status, with a focus on its application in the postoperative patient. J Neurosurg Spine 14:151-166

31. Koyfman SA, Djemil T, Burdick MJ et al (2012) Marginal recurrence requiring salvage radiotherapy after stereotactic body radiotherapy (SBRT) for spinal metastases. Int J Radiat Oncol Biol Phys 83:297-302

32. Abbatucci JS, Delozier T, Quint R, Roussel A, Brune D (1978) Radiation myelopathy of the cervical spinal cord: time, dose and volume factors. Int J Radiat Oncol Biol Phys 4:239-248

33. Kirkpatrick JP, van der Kogel AJ, Schultheiss TE (2010) Radiation dose-volume effects in the spinal cord. Int J Radiat Oncol Biol Phys 76:S42-S49

34. Sahgal A, Ma L, Gibbs I et al (2010) Spinal cord tolerance for stereotactic body radiotherapy. Int $\mathrm{J}$ Radiat Oncol Biol Phys 77:548-553

35. Sahgal A, Ma L, Weinberg V et al (2010) Reirradiation human spinal cord tolerance for stereotactic body radiotherapy. Int $\mathrm{J}$ Radiat Oncol Biol Phys 82:107-116

36. Rose PS, Laufer I, Boland PJ et al (2009) Risk of fracture after single fraction image-guided intensity-modulated radiation therapy to spinal metastases. J Clin Oncol 27:5075-5079
37. Boehling NS, Grosshans DR, Allen PK et al (2012) Vertebral compression fracture risk after stereotactic body radiotherapy for spinal metastases. J Neurosurg Spine 16:397-386

38. Milker-Zabel S, Zabel A, Thilmann C et al (2003) Clinical results of retreatment of vertebral bone metastases by stereotactic conformal radiotherapy and intensity-modulated radiotherapy. Int $\mathrm{J}$ Radiat Oncol Biol Phys 55:162-167

39. Radiation Therapy Oncology Group (RTOG) of the American College of Radiology (ACR) (2012) RTOG 0631 phase II/III study of image-guided radiosurgery/SBRT for localized spine metastasis

40. Yamada Y, Lovelock DM, Yenice KM et al (2005) Multifractionated image-guided and stereotactic intensity-modulated radiotherapy of paraspinal tumors: a preliminary report. Int J Radiat Oncol Biol Phys 62:53-61

41. Shiu AS, Chang EL, Ye J-S et al (2003) Near simultaneous computed tomography image-guided stereotactic spinal radiotherapy: an emerging paradigm for achieving true stereotaxy. Int $\mathrm{J}$ Radiat Oncol Biol Phys 57:605-613

42. Mahadevan A, Floyd S, Wong E et al (2010) Stereotactic body radiotherapy reirradiation for recurrent epidural spinal metastases. Int J Radiat Oncol Biol Phys 81:1500-1505

43. Ryu S, Rock J, Jain R et al (2010) Radiosurgical decompression of metastatic epidural compression. Cancer 116:2250-2257 